\title{
Colors and taxonomy of Centaurs and trans-Neptunian objects ${ }^{\star}$
} \author{
A. Doressoundiram ${ }^{1}$, and C. de Bergh ${ }^{1}$ \\ ${ }^{1}$ LESIA, Observatoire de Paris, 5 Place Jules Janssen, 92195 Meudon Principal Cedex, France \\ e-mail: davide.perna@obspm.fr \\ 2 Università di Roma Tor Vergata, Italy \\ INAF-Osservatorio Astronomico di Roma, Italy \\ Université Paris Diderot - Paris 7, France \\ European Southern Observatory, Chile \\ ${ }^{6}$ Department of Astronomy, University of Maryland, USA
}

D. Perna ${ }^{1,2,3}$, M. A. Barucci ${ }^{1}$, S. Fornasier ${ }^{1,4}$, F. E. DeMeo ${ }^{1}$, A. Alvarez-Candal ${ }^{1,5}$, F. Merlin ${ }^{1,6}$, E. Dotto ${ }^{3}$,

Received 12 November 2009 / Accepted 30 November 2009

\section{ABSTRACT}

\begin{abstract}
Context. The study of the surface properties of Centaurs and trans-Neptunian objects (TNOs) provides essential information about the early conditions and evolution of the outer Solar System. Due to the faintness of most of these distant and icy bodies, photometry currently constitutes the best technique to survey a statistically significant number of them.

Aims. Our aim is to investigate color properties of a large sample of minor bodies of the outer Solar System, and set their taxonomic classification.

Methods. We carried out visible and near-infrared photometry of Centaurs and TNOs, making use, respectively, of the FORS2 and ISAAC instruments at the Very Large Telescope (European Southern Observatory). Using G-mode analysis, we derived taxonomic classifications according to the Barucci et al. (2005a, AJ, 130, 1291) system.

Results. We report photometric observations of 31 objects, 10 of them have their colors reported for the first time ever. 28 Centaurs and TNOs have been assigned to a taxon.

Conclusions. We combined the entire sample of 38 objects taxonomically classified in the framework of our programme (28 objects from this work; 10 objects from DeMeo et al. 2009a, A\&A, 493, 283) with previously classified TNOs and Centaurs, looking for correlations between taxonomy and dynamics. We compared our photometric results to literature data, finding hints of heterogeneity for the surfaces of 4 objects.
\end{abstract}

Key words. Kuiper Belt: general - techniques: photometric - infrared: planetary systems

\section{Introduction}

The investigation of the surface properties of the minor bodies in the outer Solar System constitutes a major topic in modern planetary science, since they represent the "vestiges" of the leftover planetesimals from the early accretional phases of the outer proto-planetary disk. Even though they are affected by space weathering and collisional evolution (see, e.g., Hudson et al. 2008; and Leinhardt et al. 2008), they present the most pristine material in present times available for our studies, from which we can learn about the origin and early evolution of the Solar System at large distances from the Sun.

Because of the faintness of trans-Neptunian objects (TNOs) and Centaurs, spectroscopic observations (which could provide the most detailed information about their surface compositions) are feasible only for a small number of them, even when using the largest ground-based telescopes (see Barucci et al. 2008, for a recent review). Hence photometry is still the best tool to investigate the surface properties of a significant sample of these objects, allowing a global view of the whole known population.

To date photometric surveys have observed more than 200 objects. Statistical analyses were performed to search for possible correlations between colors and physical and orbital

* Based on observations carried out at the European Southern Observatory (ESO), Chile (Programmes 178.C-0036 and 178.C-0867). parameters (see Doressoundiram et al. 2008 and Tegler et al. 2008 for recent reviews). As the major result, a clustering of "cold" (low eccentricity, low inclination) classical TNOs (see Gladman et al. 2008, for a dynamical classification of objects in the outer Solar System) with very red colors was found.

Even if photometric colors cannot provide firm constraints on the surface composition, since they depend also on scattering effects in particulate regoliths and viewing geometry, they can be used to classify the objects in different groups that reasonably indicate different composition and/or evolutional history. A new TNO taxonomy (Barucci et al. 2005a; Fulchignoni et al. 2008) based on color indices $(B-V, V-R, V-I, V-J, V-H$, and $V-K$ ) identifies four classes with increasingly red colors: $\mathrm{BB}$ (neutral color), BR, IR, and RR (very red).

An ESO (European Southern Observatory) large programme devoted to the study of TNOs and Centaurs, by means of different techniques, was lead by Barucci in 2006-2008. In this framework, visible and near-infrared (NIR) photometry of a total of 45 objects was performed. The results from data acquired between October 2006 and September 2007 were published in DeMeo et al. (2009a). In this paper we present all the photometric observations executed during the second year of the large programme (November 2007-November 2008), regarding 31 objects. For 28 of these targets we were able to determine the Barucci et al. (2005a) taxonomic classification, via the G-mode 
statistical method presented in Fulchignoni et al. (2000), and to compare our results with literature data whenever available.

An analysis of the entire sample (151 objects) of taxonomically classified TNOs and Centaurs has been performed, searching for correlations between dynamical properties and taxonomy.

\section{Observations and data reduction}

All of the data presented in this work were obtained with the ESO Unit Telescope 1 (Antu) of the Very Large Telescope (VLT), located in Cerro Paranal, Chile.

The observational circumstances are reported in Table 1.

\subsection{Visible}

Visible photometry was performed with the FORS2 instrument (Appenzeller et al. 1998), equipped with a mosaic of two $2000 \times$ 4000 MIT CCD with square $15 \mu \mathrm{m}$ pixels. The observations were carried out with the standard resolution (SR) collimator and a $2 \times 2$ binning, yielding a resolution of $0.25 \mathrm{arcsec} / \mathrm{pixel}$. We used the broadband $V, R, I$ filters, centered at $0.554,0.655$ and $0.768 \mu \mathrm{m}$, respectively, adjusting the exposure time according to the object magnitude.

The images were reduced using standard procedures with the MIDAS software package: after subtraction of the bias from the raw data and flat-field correction, the instrumental magnitudes were measured via aperture photometry, with an integrating radius typically about three times the average seeing and sky subtraction performed using a 5-10 pixel wide annulus around each object. The aperture correction method (see, e.g., Barucci et al. 2000) was used for only a few cases (faint target and/or nearby field stars) to determine the object flux. The absolute calibration of the magnitudes was obtained by means of the observation of several Landolt (1992) standard fields.

\subsection{Near-infrared}

NIR photometry was performed with the ISAAC instrument (Moorwood et al. 1998), in SWI1 (short wavelength imaging) mode, using the $1024 \times 1024$ Hawaii Rockwell array with a pixel size of $18.5 \mu \mathrm{m}$ and a scale of 0.148 arcsec/pixel. We observed with the $J, H, K$ s filters, with central wavelength of $1.25,1.65$ and $2.16 \mu \mathrm{m}$, respectively. As is typical for NIR observations, the expositions were split in several images with short exposure times, in order to minimize the sky background noise.

The data were pre-reduced (dark subtraction, flat-field correction, bad pixel cleaning, sky subtraction, recombination of the images) by using the ESO ISAAC pipeline. Then, as for the visible images, target fluxes were mostly measured (with MIDAS) using classical photometry methods with apertures determined by the seeing and growth curves of the objects, reserving the aperture correction method to a few cases. To calibrate the instrumental magnitudes, standard stars from different catalogues (Persson et al. 1998; Hawarden et al. 2001) were observed.

\section{Results}

During the second year of our ESO large programme we obtained visible and NIR photometric measurements for $31 \mathrm{ob}-$ jects, 10 of them have their colors reported for the first time ever.
Table 1. Observational circumstances.

\begin{tabular}{|c|c|c|c|c|}
\hline Object & Date & $\Delta(\mathrm{AU})$ & $r(\mathrm{AU})$ & $\alpha(\mathrm{deg})$ \\
\hline (5145) Pholus & 12 Apr. 2008 & 21.212 & 21.864 & 2.0 \\
\hline \multirow[t]{2}{*}{ (10199) Chariklo } & 3 Feb. 2008 & 13.311 & 13.395 & 4.2 \\
\hline & 4 Feb. 2008 & 13.296 & 13.395 & 4.2 \\
\hline (20000) Varuna & 4 Dec. 2007 & 42.605 & 43.391 & 0.8 \\
\hline (42301) $2001 \mathrm{UR}_{163}$ & 5 Dec. 2007 & 49.625 & 50.308 & 0.8 \\
\hline (42355) Typhon & 12 Apr. 2008 & 16.892 & 17.650 & 2.2 \\
\hline \multirow[t]{2}{*}{ (44594) $1999 \mathrm{OX}_{3}$} & 21 Sep. 2008 & 22.025 & 22.889 & 1.3 \\
\hline & 22 Sep. 2008 & 22.033 & 22.888 & 1.3 \\
\hline \multirow[t]{2}{*}{ (52872) Okyrhoe } & 3 Feb. 2008 & 4.883 & 5.800 & 3.9 \\
\hline & 4 Feb. 2008 & 4.877 & 5.800 & 3.7 \\
\hline (55576) Amycus & 12 Apr. 2008 & 15.205 & 16.056 & 1.9 \\
\hline (55637) $2002 \mathrm{UX}_{25}$ & 6 Dec. 2007 & 41.263 & 41.975 & 0.9 \\
\hline \multirow{4}{*}{ (55638 $2002 \mathrm{VE}_{95}$} & 5 Dec. 2007 & 27.297 & 28.248 & 0.5 \\
\hline & 6 Dec. 2007 & 27.301 & 28.249 & 0.6 \\
\hline & 22 Nov. 2008 & 27.379 & 28.341 & 0.5 \\
\hline & 23 Nov. 2008 & 27.378 & 28.341 & 0.4 \\
\hline (73480) $2002 \mathrm{PN}_{34}$ & 10 Nov. 2007 & 14.894 & 15.344 & 3.3 \\
\hline \multirow[t]{2}{*}{ (90377) Sedna } & 21 Sep. 2008 & 87.416 & 88.015 & 0.5 \\
\hline & 22 Sep. 2008 & 87.402 & 88.014 & 0.5 \\
\hline \multirow[t]{2}{*}{ (90482) Orcus } & 3 Feb. 2008 & 46.904 & 47.807 & 0.5 \\
\hline & 4 Feb. 2008 & 46.900 & 47.807 & 0.5 \\
\hline \multirow[t]{3}{*}{ (120061) $2003 \mathrm{CO}_{1}$} & 4 Feb. 2008 & 10.937 & 11.080 & 5.1 \\
\hline & 12 Apr. 2008 & 10.179 & 11.123 & 1.8 \\
\hline & 13 Apr. 2008 & 10.175 & 11.123 & 1.8 \\
\hline (120132) $2003 \mathrm{FY}_{128}$ & 12 Apr. 2008 & 37.477 & 38.454 & 0.3 \\
\hline (120178) $2003 \mathrm{OP}_{32}$ & 21 Sep. 2008 & 40.546 & 41.365 & 0.8 \\
\hline \multirow{2}{*}{ (120348) $2004 \mathrm{TY}_{364}$} & 22 Nov. 2008 & 38.840 & 39.591 & 0.9 \\
\hline & 23 Nov. 2008 & 38.849 & 39.591 & 1.0 \\
\hline (136199) Eris & 7 Dec. 2007 & 49.652 & 50.310 & 0.8 \\
\hline \multirow[t]{4}{*}{ (144897) $2004 \mathrm{UX}_{10}$} & 4 Dec. 2007 & 38.145 & 38.837 & 1.0 \\
\hline & 5 Dec. 2007 & 38.158 & 38.837 & 1.1 \\
\hline & 6 Dec. 2007 & 38.170 & 38.837 & 1.1 \\
\hline & 23 Nov. 2008 & 38.059 & 38.879 & 0.8 \\
\hline \multirow[t]{2}{*}{ (145451) $2005 \mathrm{RM}_{43}$} & 4 Dec. 2007 & 34.298 & 35.195 & 0.7 \\
\hline & 7 Dec. 2007 & 34.316 & 35.196 & 0.7 \\
\hline \multirow[t]{2}{*}{ (145453) $2005 \mathrm{RR}_{43}$} & 4 Dec. 2007 & 37.623 & 38.511 & 0.6 \\
\hline & 7 Dec. 2007 & 37.642 & 38.512 & 0.7 \\
\hline \multirow[t]{2}{*}{ (174567) $2003 \mathrm{MW}_{12}$} & 12 Apr. 2008 & 47.314 & 47.968 & 0.9 \\
\hline & 13 Apr. 2008 & 47.302 & 47.968 & 0.9 \\
\hline \multirow[t]{2}{*}{ (208996) $2003 \mathrm{AZ}_{84}$} & 22 Nov. 2008 & 44.879 & 45.458 & 1.0 \\
\hline & 23 Nov. 2008 & 44.865 & 45.457 & 1.0 \\
\hline \multirow[t]{2}{*}{$2002 \mathrm{KY}_{14}$} & 21 Sep. 2008 & 7.802 & 8.649 & 3.8 \\
\hline & 22 Sep. 2008 & 7.810 & 8.649 & 3.8 \\
\hline \multirow[t]{2}{*}{$2003 \mathrm{UZ}_{117}$} & 22 Nov. 2008 & 38.420 & 39.368 & 0.4 \\
\hline & 23 Nov. 2008 & 38.423 & 39.367 & 0.4 \\
\hline \multirow[t]{4}{*}{$2003 \mathrm{UZ}_{413}$} & 4 Dec. 2007 & 41.171 & 42.004 & 0.7 \\
\hline & 21 Sep. 2008 & 41.466 & 42.163 & 1.0 \\
\hline & 22 Nov. 2008 & 41.276 & 42.197 & 0.5 \\
\hline & 23 Nov. 2008 & 41.282 & 42.198 & 0.5 \\
\hline \multirow[t]{2}{*}{$2007 \mathrm{UK}_{126}$} & 21 Sep. 2008 & 45.131 & 45.618 & 1.1 \\
\hline & 22 Sep. 2008 & 45.117 & 45.617 & 1.1 \\
\hline $2007 \mathrm{UM}_{126}$ & 21 Sep. 2008 & 10.202 & 11.163 & 1.5 \\
\hline & 22 Sep. 2008 & 10.199 & 11.165 & 1.5 \\
\hline $2007 \mathrm{VH}_{305}$ & 22 Nov. 2008 & 7.854 & 8.638 & 4.2 \\
\hline & 23 Nov. 2008 & 7.863 & 8.636 & 4.3 \\
\hline $2008 \mathrm{FC}_{76}$ & 20 Sep. 2008 & 10.968 & 11.690 & 3.5 \\
\hline & 21 Sep. 2008 & 10.976 & 11.688 & 3.6 \\
\hline $2008 \mathrm{SJ}_{236}$ & 22 Nov. 2008 & 5.522 & 6.364 & 5.0 \\
\hline & 23 Nov. 2008 & 5.530 & 6.363 & 5.1 \\
\hline
\end{tabular}

Notes. $\Delta, r$ and $\alpha$ are the topocentric distance, the heliocentric distance, and the phase angle, respectively.

Table 2 lists the resulting magnitude values, as well as the computed absolute magnitudes $H$ of the targets, calculated as

$H=V-5 \log (r \Delta)-\alpha \beta$, 
Table 2. Observed magnitudes.

\begin{tabular}{|c|c|c|c|c|c|c|c|c|c|}
\hline Object & Date & $\mathrm{UT}_{\mathrm{START}}$ & V & $R$ & I & $J$ & $H$ & $K_{\mathrm{s}}$ & $H_{V}$ \\
\hline (5145) Pholus & 12 Apr. 2008 & $05: 48$ & $21.33 \pm 0.09$ & $20.64 \pm 0.09$ & $19.95 \pm 0.15$ & $\ldots$ & $\ldots$ & $\ldots$ & $7.78 \pm 0.09$ \\
\hline \multirow[t]{2}{*}{ (10199) Chariklo ${ }^{a}$} & 3 Feb. 2008 & 07:41 & $\ldots$ & $\ldots$ & $\ldots$ & $17.11 \pm 0.06$ & $16.61 \pm 0.05$ & $16.38 \pm 0.06$ & $\ldots$ \\
\hline & 4 Feb. 2008 & 07:41 & $18.79 \pm 0.02$ & $18.34 \pm 0.02$ & $17.88 \pm 0.03$ & $\ldots$ & $\ldots$ & $\ldots$ & $7.07 \pm 0.04$ \\
\hline \multirow[t]{2}{*}{ (20000) Varuna } & 4 Dec. 2007 & 06:09 & $20.49 \pm 0.03$ & $19.88 \pm 0.03$ & $\ldots$ & $\ldots$ & $\ldots$ & $\ldots$ & $4.04 \pm 0.04$ \\
\hline & 4 Dec. 2007 & $06: 12$ & $20.46 \pm 0.04$ & $19.88 \pm 0.03$ & $\ldots$ & $\ldots$ & $\ldots$ & $\ldots$ & $4.01 \pm 0.05$ \\
\hline \multirow[t]{2}{*}{ (42301) $2001 \mathrm{UR}_{163}$} & 5 Dec. 2007 & $00: 52$ & $21.82 \pm 0.06$ & $20.98 \pm 0.07$ & $\ldots$ & $\ldots$ & $\ldots$ & $\ldots$ & $4.72 \pm 0.06$ \\
\hline & 5 Dec. 2007 & 00:55 & $21.81 \pm 0.05$ & $20.98 \pm 0.07$ & $\ldots$ & $\ldots$ & $\ldots$ & $\ldots$ & $4.71 \pm 0.06$ \\
\hline (42355) Typhon ${ }^{b}$ & 12 Apr. 2008 & 00:59 & $20.50 \pm 0.08$ & $20.00 \pm 0.08$ & $19.70 \pm 0.08$ & $\ldots$ & $\ldots$ & $\ldots$ & $7.77 \pm 0.11$ \\
\hline \multirow[t]{2}{*}{ (44594) 1999 OX $_{3}$} & 21 Sep. 2008 & 04:07 & $21.26 \pm 0.05$ & $20.54 \pm 0.06$ & $19.96 \pm 0.07$ & $\ldots$ & $\ldots$ & $\ldots$ & $7.57 \pm 0.06$ \\
\hline & 22 Sep. 2008 & $04: 49$ & $\ldots$ & $\ldots$ & $\ldots$ & $19.08 \pm 0.12$ & $18.75 \pm 0.08$ & $18.69 \pm 0.08$ & $\ldots$ \\
\hline \multirow[t]{2}{*}{ (52872) Okyrhoe ${ }^{c}$} & 3 Feb. 2008 & $06: 41$ & $\ldots$ & $\ldots$ & $\ldots$ & $16.84 \pm 0.06$ & $16.39 \pm 0.07$ & $16.28 \pm 0.05$ & $\ldots$ \\
\hline & 4 Feb. 2008 & $06: 18$ & $18.63 \pm 0.02$ & $18.14 \pm 0.02$ & $17.64 \pm 0.02$ & $\ldots$ & $\ldots$ & $\ldots$ & $10.97 \pm 0.04$ \\
\hline (55576) Amycus & 12 Apr. 2008 & $03: 33$ & $20.42 \pm 0.08$ & $19.78 \pm 0.08$ & $19.20 \pm 0.15$ & $\ldots$ & $\ldots$ & $\ldots$ & $8.27 \pm 0.08$ \\
\hline (55637) $2002 \mathrm{UX}_{25}$ & 6 Dec. 2007 & 01:19 & $\ldots$ & $\ldots$ & $\ldots$ & $18.55 \pm 0.03$ & $18.25 \pm 0.04$ & $18.21 \pm 0.06$ & $\ldots$ \\
\hline \multirow[t]{5}{*}{ (55638) $2002 \mathrm{VE}_{95}$} & 5 Dec. 2007 & $03: 35$ & $20.31 \pm 0.03$ & $19.59 \pm 0.04$ & $\ldots$ & $\ldots$ & $\ldots$ & $\ldots$ & $5.80 \pm 0.03$ \\
\hline & 5 Dec. 2007 & 03:38 & $20.31 \pm 0.03$ & $19.59 \pm 0.04$ & $\ldots$ & $\ldots$ & $\ldots$ & $\ldots$ & $5.80 \pm 0.03$ \\
\hline & 6 Dec. 2007 & 02:09 & $\ldots$ & $\ldots$ & $\ldots$ & $18.11 \pm 0.04$ & $17.78 \pm 0.04$ & $17.74 \pm 0.04$ & $\ldots$ \\
\hline & 22 Nov. 2008 & $05: 36$ & $20.28 \pm 0.06$ & $19.53 \pm 0.08$ & $18.77 \pm 0.09$ & $\ldots$ & $\ldots$ & $\ldots$ & $5.76 \pm 0.06$ \\
\hline & 23 Nov. 2008 & $05: 57$ & $\ldots$ & $\ldots$ & $\ldots$ & $18.04 \pm 0.07$ & $17.68 \pm 0.07$ & $17.63 \pm 0.09$ & $\ldots$ \\
\hline (73480) $2002 \mathrm{PN}_{34}{ }^{c}$ & 10 Nov. 2007 & $00: 36$ & $20.68 \pm 0.03$ & $20.25 \pm 0.05$ & $\ldots$ & $19.00 \pm 0.05$ & $18.49 \pm 0.06$ & $18.29 \pm 0.05$ & $8.42 \pm 0.10$ \\
\hline \multirow[t]{2}{*}{ (90377) Sedna } & 21 Sep. 2008 & $06: 33$ & $21.34 \pm 0.04$ & $20.57 \pm 0.05$ & $19.93 \pm 0.05$ & $\ldots$ & $\ldots$ & $\ldots$ & $1.84 \pm 0.04$ \\
\hline & 22 Sep. 2008 & $06: 57$ & $\ldots$ & $\ldots$ & $\ldots$ & $19.20 \pm 0.04$ & $18.78 \pm 0.06$ & $\ldots$ & $\ldots$ \\
\hline \multirow[t]{2}{*}{ (90482) Orcus $^{c}$} & 3 Feb. 2008 & $04: 46$ & $\ldots$ & $\ldots$ & $\ldots$ & $17.91 \pm 0.05$ & $17.72 \pm 0.07$ & $17.89 \pm 0.05$ & $\ldots$ \\
\hline & 4 Feb. 2008 & 05:04 & $19.12 \pm 0.02$ & $18.73 \pm 0.02$ & $18.37 \pm 0.02$ & $\ldots$ & $\ldots$ & $\ldots$ & $2.30 \pm 0.03$ \\
\hline \multirow[t]{2}{*}{ (120061) $2003 \mathrm{CO}_{1}$} & 4 Feb. 2008 & $08: 17$ & $19.93 \pm 0.02$ & $19.45 \pm 0.03$ & $19.01 \pm 0.03$ & $\ldots$ & $\ldots$ & $\ldots$ & $8.95 \pm 0.05$ \\
\hline & 12 Apr. 2008 & $04: 40$ & $19.63 \pm 0.08$ & $19.20 \pm 0.09$ & $18.82 \pm 0.15$ & $\ldots$ & $\ldots$ & $\ldots$ & $9.16 \pm 0.08$ \\
\hline (120132) $2003 \mathrm{FY}_{128}$ & 12 Apr. 2008 & $02: 22$ & $20.93 \pm 0.09$ & $20.34 \pm 0.09$ & $19.86 \pm 0.15$ & $\ldots$ & $\ldots$ & $\ldots$ & $5.09 \pm 0.09$ \\
\hline (120178) $2003 \mathrm{OP}_{32}$ & 21 Sep. 2008 & 02:10 & $20.25 \pm 0.03$ & $19.86 \pm 0.05$ & $19.50 \pm 0.05$ & $\ldots$ & $\ldots$ & $\ldots$ & $4.02 \pm 0.04$ \\
\hline \multirow{2}{*}{ (120348) $2004 \mathrm{TY}_{364}$} & 22 Nov. 2008 & $04: 32$ & $20.64 \pm 0.03$ & $20.04 \pm 0.04$ & $19.52 \pm 0.04$ & $\ldots$ & $\ldots$ & $\ldots$ & $4.58 \pm 0.04$ \\
\hline & 23 Nov. 2008 & $03: 22$ & $\ldots$ & $\ldots$ & $\ldots$ & $18.87 \pm 0.03$ & $18.42 \pm 0.05$ & $18.39 \pm 0.08$ & $\ldots$ \\
\hline (136199) Eris $^{d}$ & 7 Dec. 2007 & 00:20 & $\ldots$ & $\ldots$ & $\ldots$ & $17.90 \pm 0.06$ & $17.85 \pm 0.05$ & $18.15 \pm 0.06$ & $\ldots$ \\
\hline \multirow[t]{5}{*}{ (144897) $2004 \mathrm{UX}_{10}$} & 4 Dec. 2007 & $00: 53$ & $20.61 \pm 0.04$ & $20.04 \pm 0.04$ & $\ldots$ & $\ldots$ & $\ldots$ & $\ldots$ & $4.62 \pm 0.05$ \\
\hline & 4 Dec. 2007 & $00: 56$ & $20.63 \pm 0.04$ & $20.05 \pm 0.04$ & $\ldots$ & $\ldots$ & $\ldots$ & $\ldots$ & $4.64 \pm 0.05$ \\
\hline & 5 Dec. 2007 & $02: 24$ & $20.63 \pm 0.03$ & $20.06 \pm 0.04$ & $\ldots$ & $\ldots$ & $\ldots$ & $\ldots$ & $4.62 \pm 0.04$ \\
\hline & 6 Dec. 2007 & 00:39 & $\ldots$ & $\ldots$ & $\ldots$ & $18.97 \pm 0.06$ & $18.55 \pm 0.08$ & $18.55 \pm 0.09$ & $\ldots$ \\
\hline & 23 Nov. 2008 & $02: 26$ & $\ldots$ & $\ldots$ & $\ldots$ & $19.02 \pm 0.03$ & $18.60 \pm 0.04$ & $18.64 \pm 0.06$ & $\ldots$ \\
\hline \multirow[t]{3}{*}{ (145451) $2005 \mathrm{RM}_{43}$} & 4 Dec. 2007 & $03: 22$ & $20.04 \pm 0.04$ & $19.66 \pm 0.03$ & $\ldots$ & $\ldots$ & $\ldots$ & $\ldots$ & $4.53 \pm 0.05$ \\
\hline & 4 Dec. 2007 & $03: 25$ & $20.07 \pm 0.04$ & $19.66 \pm 0.03$ & $\ldots$ & $\ldots$ & $\ldots$ & $\ldots$ & $4.56 \pm 0.05$ \\
\hline & 7 Dec. 2007 & $03: 14$ & $\ldots$ & $\ldots$ & $\ldots$ & $18.95 \pm 0.04$ & $18.76 \pm 0.05$ & $18.71 \pm 0.06$ & $\ldots$ \\
\hline (145453) $2005 \mathrm{RR}_{43}$ & 4 Dec. 2007 & $02: 26$ & $20.05 \pm 0.03$ & $19.66 \pm 0.04$ & $\ldots$ & $\ldots$ & $\ldots$ & $\ldots$ & $4.16 \pm 0.03$ \\
\hline & 4 Dec. 2007 & $02: 29$ & $20.08 \pm 0.03$ & $19.66 \pm 0.04$ & $\ldots$ & $\ldots$ & $\ldots$ & $\ldots$ & $4.19 \pm 0.03$ \\
\hline & 7 Dec. 2007 & $02: 24$ & $\ldots$ & $\ldots$ & $\ldots$ & $19.28 \pm 0.04$ & $19.47 \pm 0.07$ & $19.67 \pm 0.15$ & $\ldots$ \\
\hline (174567) $2003 \mathrm{MW}_{12}$ & 12 Apr. 2008 & 07:07 & $20.57 \pm 0.08$ & $19.99 \pm 0.08$ & $19.57 \pm 0.15$ & $\ldots$ & $\ldots$ & $\ldots$ & $3.66 \pm 0.08$ \\
\hline (208996) $2003 \mathrm{AZ}_{84}$ & 22 Nov. 2008 & 06:07 & $20.46 \pm 0.03$ & $20.08 \pm 0.04$ & $19.71 \pm 0.03$ & $\ldots$ & $\ldots$ & $\ldots$ & $3.77 \pm 0.04$ \\
\hline & 23 Nov. 2008 & $07: 10$ & $\ldots$ & $\ldots$ & $\ldots$ & $19.26 \pm 0.04$ & $18.92 \pm 0.07$ & $\ldots$ & $\ldots$ \\
\hline $2002 \mathrm{KY}_{14}$ & 21 Sep. 2008 & $00: 56$ & $19.93 \pm 0.02$ & $19.23 \pm 0.03$ & $18.58 \pm 0.04$ & $\ldots$ & $\ldots$ & $\ldots$ & $10.37 \pm 0.04$ \\
\hline & 22 Sep. 2008 & $02: 27$ & $\ldots$ & $\ldots$ & $\ldots$ & $17.85 \pm 0.06$ & $17.56 \pm 0.07$ & $17.54 \pm 0.08$ & $\ldots$ \\
\hline $2003 \mathrm{UZ}_{117}$ & 22 Nov. 2008 & $03: 21$ & $21.13 \pm 0.03$ & $20.77 \pm 0.04$ & $20.43 \pm 0.04$ & $\ldots$ & $\ldots$ & $\ldots$ & $5.18 \pm 0.03$ \\
\hline & 23 Nov. 2008 & 05:08 & $\ldots$ & $\ldots$ & $\ldots$ & $20.41 \pm 0.09$ & $20.63 \pm 0.17$ & $\ldots$ & $\ldots$ \\
\hline $2003 \mathrm{UZ}_{413}$ & 4 Dec. 2007 & $04: 28$ & $20.70 \pm 0.04$ & $20.22 \pm 0.04$ & $\ldots$ & $\ldots$ & $\ldots$ & $\ldots$ & $4.41 \pm 0.05$ \\
\hline & 4 Dec. 2007 & $04: 31$ & $20.67 \pm 0.04$ & $20.22 \pm 0.04$ & $\ldots$ & $\ldots$ & $\ldots$ & $\ldots$ & $4.38 \pm 0.05$ \\
\hline & 21 Sep. 2008 & $05: 31$ & $20.71 \pm 0.04$ & $20.25 \pm 0.05$ & $19.88 \pm 0.06$ & $\ldots$ & $\ldots$ & $\ldots$ & $4.36 \pm 0.05$ \\
\hline & 22 Nov. 2008 & $03: 33$ & $20.63 \pm 0.03$ & $20.22 \pm 0.04$ & $19.82 \pm 0.04$ & $\ldots$ & $\ldots$ & $\ldots$ & $4.36 \pm 0.03$ \\
\hline & 23 Nov. 2008 & 04:03 & $\ldots$ & $\ldots$ & $\ldots$ & $19.29 \pm 0.05$ & $18.92 \pm 0.09$ & $18.77 \pm 0.09$ & $\ldots$ \\
\hline $2007 \mathrm{UK}_{126}$ & 21 Sep. 2008 & 08:05 & $20.41 \pm 0.03$ & $19.79 \pm 0.04$ & $19.32 \pm 0.04$ & $\ldots$ & $\ldots$ & $\ldots$ & $3.69 \pm 0.04$ \\
\hline & 22 Sep. 2008 & $07: 43$ & $\ldots$ & $\ldots$ & $\ldots$ & $18.88 \pm 0.07$ & $18.52 \pm 0.08$ & $\ldots$ & $\ldots$ \\
\hline $2007 \mathrm{UM}_{126}$ & 21 Sep. 2008 & $04: 26$ & $20.88 \pm 0.03$ & $20.44 \pm 0.03$ & $20.00 \pm 0.03$ & $\ldots$ & $\ldots$ & $\ldots$ & $10.43 \pm 0.03$ \\
\hline & 22 Sep. 2008 & $05: 57$ & $\ldots$ & $\ldots$ & $\ldots$ & $\ldots$ & $18.84 \pm 0.13$ & $18.50 \pm 0.08$ & $\ldots$ \\
\hline $2007 \mathrm{VH}_{305}$ & 22 Nov. 2008 & $02: 14$ & $21.44 \pm 0.04$ & $20.96 \pm 0.04$ & $20.48 \pm 0.05$ & $\ldots$ & $\ldots$ & $\ldots$ & $11.82 \pm 0.06$ \\
\hline & 23 Nov. 2008 & $00: 38$ & $\ldots$ & $\ldots$ & $\ldots$ & $19.70 \pm 0.10$ & $19.06 \pm 0.12$ & $19.43 \pm 0.15$ & $\ldots$ \\
\hline $\mathbf{2 0 0 8} \mathbf{F} \mathbf{C}_{76}$ & 20 Sep. 2008 & $23: 55$ & $20.38 \pm 0.03$ & $19.67 \pm 0.05$ & $19.04 \pm 0.06$ & $\ldots$ & $\ldots$ & $\ldots$ & $9.46 \pm 0.05$ \\
\hline & 21 Sep. 2008 & $23: 53$ & $\ldots$ & $\ldots$ & $\ldots$ & $18.40 \pm 0.08$ & $18.00 \pm 0.08$ & $17.88 \pm 0.09$ & $\ldots$ \\
\hline $2008 \mathbf{S J}_{236}$ & 22 Nov. 2008 & $00: 24$ & $20.75 \pm 0.03$ & $20.13 \pm 0.03$ & $19.63 \pm 0.04$ & $\ldots$ & $\ldots$ & $\ldots$ & $12.47 \pm 0.06$ \\
\hline & 23 Nov. 2008 & $01: 28$ & $\ldots$ & $\ldots$ & $\ldots$ & $19.01 \pm 0.07$ & $18.60 \pm 0.11$ & $18.83 \pm 0.12$ & $\ldots$ \\
\hline
\end{tabular}

Notes. Objects in bold have their colors reported for the first time ever.

${ }^{(a)}$ Computed magnitudes from Guilbert et al. (2009). ${ }^{(b)}$ Computed magnitudes from Alvarez-Candal et al. (2009). ${ }^{(c)}$ Computed magnitudes from DeMeo et al. (2009b). ${ }^{(d)}$ Computed magnitudes from Merlin et al. (2009). 
Table 3. Taxonomic classification.

\begin{tabular}{|c|c|c|c|c|c|}
\hline Object & Dyn. Class & Fulchignoni et al. 2008 & $\begin{array}{c}\text { Taxonomy } \\
\text { DeMeo et al. 2009a }\end{array}$ & This work & $N$ \\
\hline (5145) Pholus & Centaur & $\mathrm{RR}$ & $\ldots$ & RR & 2 \\
\hline (10199) Chariklo & Centaur & $\mathrm{BR}$ & $\mathrm{BR}, \mathrm{BB}$ & $\mathrm{BR}$ & 5 \\
\hline (42355) Typhon & Scattered & $\mathrm{BR}$ & $\mathrm{BR}$ & $\mathrm{BR}, \mathrm{BB}$ & $5^{a}$ \\
\hline (44594) $1999 \mathrm{OX}_{3}$ & Scattered & RR & $\ldots$ & $\mathrm{RR}$ & 5 \\
\hline (52872) Okyrhoe & Jupiter-coupled & BR & $\ldots$ & BR,IR & 5 \\
\hline (55576) Amycus & Centaur & RR & $\ldots$ & RR,IR & 2 \\
\hline (55637) $2002 \mathrm{UX}_{25}$ & Classical & IR & RR,IR & $\mathrm{RR}$ & $5^{b}$ \\
\hline (55638) $2002 \mathrm{VE}_{95}$ & Resonant (3:2) & RR & $\ldots$ & $\mathrm{RR}$ & 5 \\
\hline (73480) $2002 \mathrm{PN}_{34}$ & Scattered & $\ldots$ & $\ldots$ & $\mathrm{BR}, \mathrm{BB}$ & 4 \\
\hline (90377) Sedna & Detached & $\mathrm{RR}$ & $\ldots$ & $\mathrm{RR}$ & 4 \\
\hline (90482) Orcus & Resonant (3:2) & $\mathrm{BB}$ & $\ldots$ & $\mathrm{BB}$ & 5 \\
\hline (120061) $2003 \mathrm{CO}_{1}$ & Centaur & $\ldots$ & $\ldots$ & $\mathrm{BR}$ & 2 \\
\hline (120132) $2003 \mathrm{FY}_{128}$ & Detached & $\ldots$ & $\mathrm{BR}$ & $\mathrm{BR}$ & $5^{a}$ \\
\hline (120178) $2003 \mathrm{OP}_{32}$ & Classical & $\ldots$ & $\ldots$ & BB,BR & 2 \\
\hline (120348) $2004 \mathrm{TY}_{364}$ & Classical & $\ldots$ & $\ldots$ & IR,RR,BR & 5 \\
\hline (136199) Eris & Detached & $\mathrm{BB}$ & $\mathrm{BB}$ & $\mathrm{BB}$ & $5^{b}$ \\
\hline (144897) $2004 \mathrm{UX}_{10}$ & Classical & $\ldots$ & $\ldots$ & $\mathrm{BR}$ & 4 \\
\hline (145451) $2005 \mathrm{RM}_{43}$ & Detached & $\ldots$ & $\mathrm{BB}$ & $\mathrm{BB}$ & 4 \\
\hline (145453) $2005 \mathrm{RR}_{43}$ & Classical & $\ldots$ & $\mathrm{BB}$ & BB & 4 \\
\hline (174567) $2003 \mathrm{MW}_{12}$ & Classical & $\ldots$ & $\ldots$ & IR,BR,RR & 2 \\
\hline (208996) $2003 \mathrm{AZ}_{84}$ & Resonant (3:2) & $\mathrm{BB}$ & $\mathrm{BB}$ & $\mathrm{BB}$ & 4 \\
\hline $2002 \mathrm{KY}_{14}$ & Centaur & $\ldots$ & $\ldots$ & RR & 5 \\
\hline $2003 \mathrm{UZ}_{117}$ & Classical & $\ldots$ & $\ldots$ & $\mathrm{BB}$ & 4 \\
\hline $2003 \mathrm{UZ}_{413}$ & Resonant (3:2) & $\ldots$ & $\ldots$ & $\mathrm{BB}$ & 5 \\
\hline $2007 \mathrm{UK}_{126}$ & Scattered & $\ldots$ & $\ldots$ & $*$ & 4 \\
\hline $2007 \mathrm{UM}_{126}$ & Centaur & $\ldots$ & $\ldots$ & $\mathrm{BR}, \mathrm{BB}$ & 4 \\
\hline $2007 \mathrm{VH}_{305}$ & Centaur & $\ldots$ & $\ldots$ & $\mathrm{BR}$ & 5 \\
\hline $2008 \mathrm{FC}_{76}$ & Centaur & $\ldots$ & $\ldots$ & $\mathrm{RR}$ & 5 \\
\hline $2008 \mathrm{SJ}_{236}$ & Centaur & $\ldots$ & $\ldots$ & $\mathrm{RR}$ & 5 \\
\hline (28978) Ixion & Resonant (3:2) & IR & $\mathrm{BB}$ & & \\
\hline (32532) Thereus & Centaur & $\mathrm{BR}$ & $\mathrm{BB}$ & & \\
\hline (47171) $1999 \mathrm{TC}_{36}$ & Resonant (3:2) & $\mathrm{RR}$ & $\mathrm{RR}$ & & \\
\hline (47932) $2000 \mathrm{GN}_{171}$ & Resonant (3:2) & IR & BR,IR & & \\
\hline (50000) Quaoar & Classical & $\ldots$ & $\mathrm{RR}$ & & \\
\hline (54598) Bienor & Centaur & $\mathrm{BR}$ & $\mathrm{BR}$ & & \\
\hline (55565) $2002 \mathrm{AW}_{197}$ & Classical & IR & IR,RR & & \\
\hline (60558) Echeclus & Jupiter-coupled & $\mathrm{BR}$ & $\mathrm{BR}, \mathrm{BB}$ & & \\
\hline (90568) $2004 \mathrm{GV}_{9}$ & Classical & $\ldots$ & $\mathrm{BR}$ & & \\
\hline $2003 \mathrm{QW}_{90}$ & Classical & $\ldots$ & IR,RR,BR & & \\
\hline
\end{tabular}

Notes. Objects from the ESO large programme taken into account for the statistical analysis of taxa. Dynamical classes are according to Gladman et al. (2008). First 29 bodies are classified in this work, last 10 objects come from DeMeo et al. (2009a). Whenever multiple taxonomic types are possible, classes are listed by ascending deviation of the object colors from the class' averages. The symbol * indicates that the object did not fall within any of the four taxonomic classes. $N$ is the number of colors we used in classifying each object.

(a) Using near-infrared data from DeMeo et al. (2009a). ${ }^{(b)}$ Using visible data from DeMeo et al. (2009a).

where $V$ represents the visible magnitude reported in Col. 4 of Table 2, $\Delta, r$ and $\alpha$ are the topocentric and heliocentric distances and the phase angle given in Table 1 , respectively, and $\beta$ is the phase curve slope (mag/deg). For TNOs, we assumed $\beta=0.14 \pm$ $0.03 \mathrm{mag} / \mathrm{deg}$, the modal value of the measurements published by Sheppard \& Jewitt (2002). For Centaurs and Jupiter-coupled objects, we assumed $\beta=0.11 \pm 0.01 \mathrm{mag} / \mathrm{deg}$, the result of a least squares fit by Doressoundiram et al. (2005) of the linear phase function $\phi(\alpha)=10^{-\alpha \beta}$ of data from Bauer et al. (2003).
On the basis of the obtained color indices, the taxonomic classification of the targets was derived via the G-mode statistical method presented in Fulchignoni et al. (2000), using the taxonomy for TNOs and Centaurs introduced by Barucci et al. (2005a). This taxonomy identifies four classes, that reasonably indicate different composition and/or evolutional history, with increasingly red colors: BB (neutral colors with respect to the Sun), BR, IR, RR (very red colors). We applied the abovementioned algorithm to objects for which two or more color 
indices were available (i.e., to 29 out of the 31 observed TNOs and Centaurs). We classified each object whenever its colors were within $3 \sigma$ of the class' average values. Obviously, a higher number of available colors implies a better reliability of the class determination. In cases where more than one class is within $3 \sigma$, we assigned a multiple designation to the object, with taxonomic classes ordered by ascending deviation of its colors from the class' averages. The taxonomic designations are reported in Table 3, along with the dynamical classification of the objects (according to Gladman et al. 2008).

We classified 28 objects: seven of them turned out to belong to the BB class, five were BR, eight were RR. The remaining eight targets got a multiple designation. Whenever a previous classification was available in the literature, a consistent result was obtained, even for objects with only 2 colors.

Only $2007 \mathrm{UK}_{126}$, even with four color measurements, did not fall within any class of the existing taxonomy. Indeed, according to its visible colors an IR, RR classification could be derived, while its infrared colors match those of a BB, BR object. Interestingly, the same result was obtained by DeMeo et al. (2009a) for two other TNOs, (26375) $1999 \mathrm{DE}_{9}$ and (145452) $2005 \mathrm{RN}_{43}$. This fact, as well as the presence of several multiple classifications, could support the idea that further groups could be found as the number of analysed objects increases, leading to a refinement of the current taxonomy.

Below, we discuss selected objects in further detail.

(10199) Chariklo: Using five color indices, we classified this Centaur as a BR object, as did Fulchignoni et al. (2008) on the basis of the mean colors published in literature. Our results, obtained in February 2008 (and already published in Guilbert et al. 2009), agree with these average measurements except for the $V-K$ color which is about 0.2 mag redder in our dataset. Interestingly, Chariklo was already observed in the framework of our programme in March 2007 (DeMeo et al. 2009a, who assigned to Chariklo a BR, BB classification), but we find no match with these previous results. Since the acquisition and the reduction of the data were carried out in the same way in both observing runs, this is a probable indication of heterogeneity on the surface of this Centaur, as already proposed in previous works (see Guilbert et al. 2009, and references therein).

(90377) Sedna: The photometric colors and taxonomic classification (RR) we derived for Sedna are in agreement with those published by Barucci et al. (2005b), except for the $V-J$ color which is approximately 0.2 mag bluer in our case. Since images in different filters have not been acquired simultaneously, the observation of different rotational phases of the object could affect color determinations. Nevertheless the light curve of Sedna has an amplitude of only $0.02 \mathrm{mag}$ (Gaudi et al. 2005), hence different observed silhouettes of the body cannot explain the found discrepancy, which could instead be attributed to surface heterogeneity.

(120348) $2004 T_{364}$ : Even if a triple designation (IR, RR, BR) has been assigned to this object, we note that the IR classification is strongly favored.

(145451) $2005 R M_{43}$ : The new data presented here confirm the BB classification already obtained in the framework of our large programme, even if we found both $V-J$ and $V-H$ colors $\sim 0.2$ mag redder than in DeMeo et al. (2009a), while the $V-K$ color is consistent. Observations with different filters have not been carried out simultaneously, but, as for Sedna, the

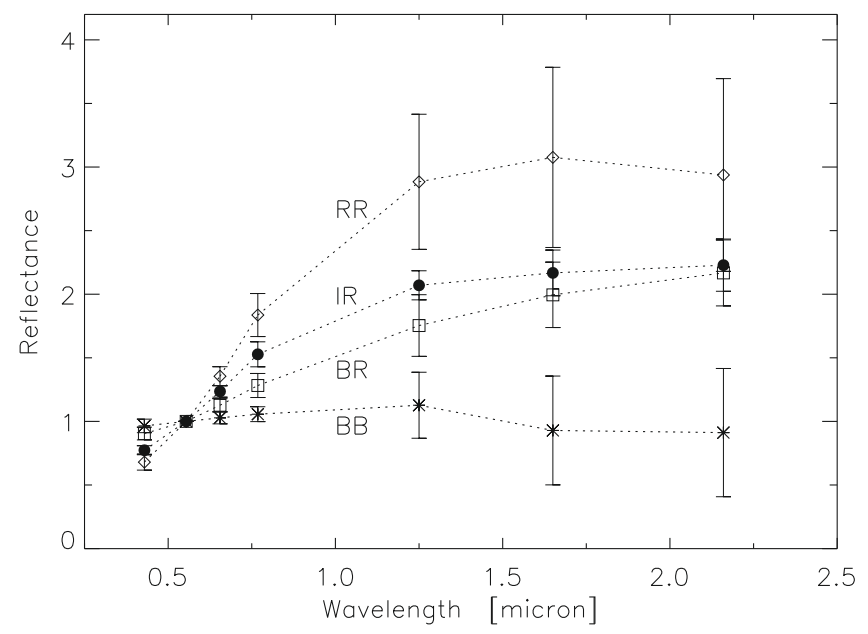

Fig. 1. Average reflectance values for each taxon, normalized to the Sun and to the $V$ colors.

amplitude of the light curve is smaller than the observed discrepancies $(\Delta m=0.12 \pm 0.05 \mathrm{mag}$; Perna et al. 2009), suggesting possible surface heterogeneity.

(145453) $2005 R R_{43}$ : We confirm that $2005 R^{2} R_{43}$ is a BB object, as classified by DeMeo et al. (2009a), but our $V-H$ color is 0.4 mag bluer than published by the same authors. Again, images with different filters have not been acquired at the same moment but the amplitude of the light curve is only $\Delta m=0.12 \pm 0.03 \mathrm{mag}$ (Perna et al. 2009), so the observation of different compositions on the surface is a likely explanation for the reported discrepancy.

(174567) $2003 M W_{12}$ : Although this object was classified as IR, $\mathrm{BR}, \mathrm{RR}$ by our analysis, as for $2004 \mathrm{TY}_{364}$ the IR designation is strongly favored.

\section{Statistical analysis}

In the framework of our ESO large programme we derived the taxonomy of 38 objects ( 28 objects from this work plus 10 objects from DeMeo et al. 2009a; see Table 3). Nineteen of them have been classified for the first time, while 4 targets were assigned different classes with respect to the results by Fulchignoni et al. (2008), who classified all of the 133 TNOs and Centaurs for which data from the literature were available before our observations.

Considering a total sample of 151 objects (because 1998 $\mathrm{WU}_{24}$ has an unusual orbit, it is not a Centaur, and therefore is not considered in this analysis), we obtained the average colors of each taxon, reported in Table 4 and represented in Fig. 1 as reflectance values normalized to the Sun and to the $V$ colors. In the cases where multiple taxonomic classes were assigned to an object, we took into account only the first designation. Then, we analysed the distribution of the four taxonomic groups with respect to the dynamical properties of the objects.

First of all, we verified the sampling of the taxa within each dynamical class (Fig. 2). The well-known color bimodality of Centaurs (see, e.g., Peixinho et al. 2003) clearly emerges, since 13 out of 25 objects belong to the BR group, while 10 of them fall in the RR class. All of the four new IR-classified objects are classical TNOs, confirming the finding that IR objects seem to be concentrated in the resonant and classical dynamical classes, as stated by Fulchignoni et al. (2008). As reported by the same 
Table 4. Average colors of the four taxa.

\begin{tabular}{lcccccc}
\hline \hline Class & $B-V$ & $V-R$ & $V-I$ & $V-J$ & $V-H$ & $V-K$ \\
\hline BB & $0.68 \pm 0.06$ & $0.39 \pm 0.05$ & $0.75 \pm 0.06$ & $1.20 \pm 0.25$ & $1.28 \pm 0.50$ & $1.32 \pm 0.60$ \\
BR & $0.75 \pm 0.06$ & $0.49 \pm 0.05$ & $0.96 \pm 0.08$ & $1.68 \pm 0.15$ & $2.11 \pm 0.14$ & $2.26 \pm 0.13$ \\
IR & $0.92 \pm 0.05$ & $0.59 \pm 0.04$ & $1.15 \pm 0.07$ & $1.86 \pm 0.06$ & $2.20 \pm 0.09$ & $2.29 \pm 0.10$ \\
RR & $1.06 \pm 0.10$ & $0.69 \pm 0.06$ & $1.35 \pm 0.10$ & $2.22 \pm 0.20$ & $2.58 \pm 0.25$ & $2.59 \pm 0.28$ \\
\hline
\end{tabular}

Notes. All of the 151 Centaurs and TNOs that have been classified thus far are taken into account. In the cases where multiple taxonomic classes were assigned to an object, we considered the first designation.

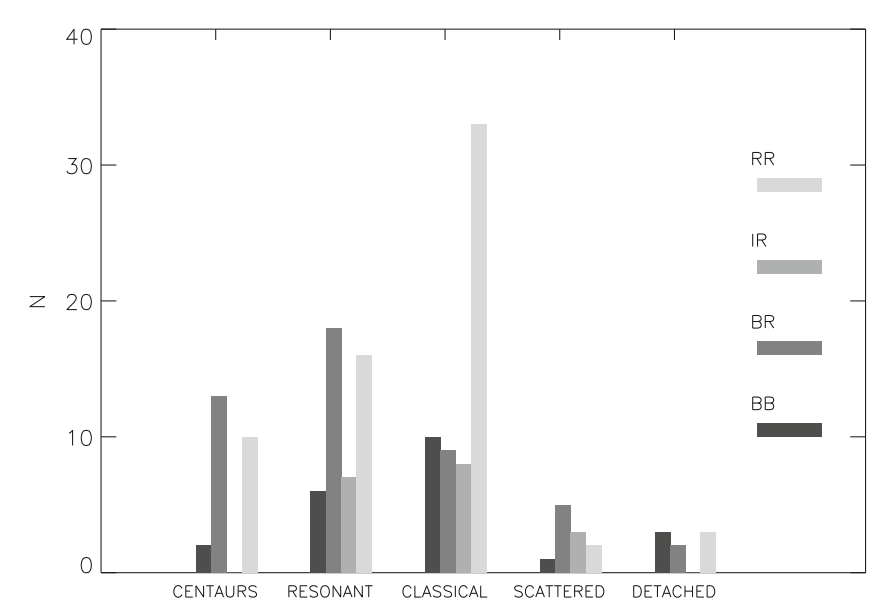

Fig. 2. Distribution of the taxa within each dynamical class, as defined by Gladman et al. (2008).

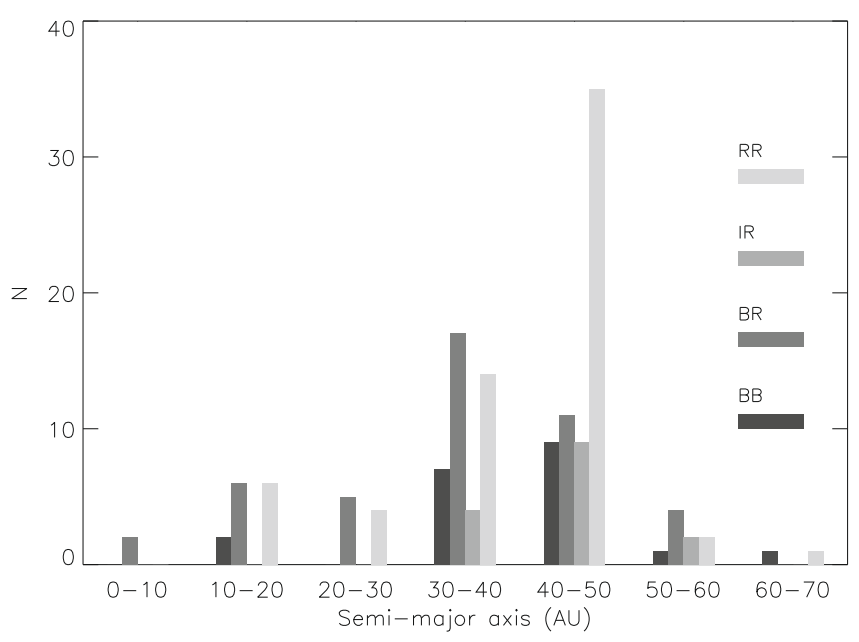

Fig. 3. Distribution of the taxa with respect to the semimajor axis of the objects. A 10 AU binning is adopted.

authors, RR objects dominate the classical TNOs. Our new results, however, do not conform to this behavior, as a quite equal division of the four taxa appears among the classical TNOs in the objects constituting the large programme sample.

In Fig. 3 we present the distribution of taxonomical classes with respect to the semimajor axes $a$ of the objects. A $10 \mathrm{AU}$ binning is adopted (nine objects are out of the scale). As already noted by Fulchignoni et al. (2008), the more distant TNOs belong to all the four taxa in a quite uniform way, while for $a \lessgtr 30^{\circ}$ the BR and RR classes dominate the population.

Finally, Fig. 4 reports the distribution of the taxa with respect to the orbital inclination $i$. A $5^{\circ}$ binning is adopted (two objects are out of the scale). Inclinations of RR-types are quite low, in agreement with the previously mentioned finding of a red

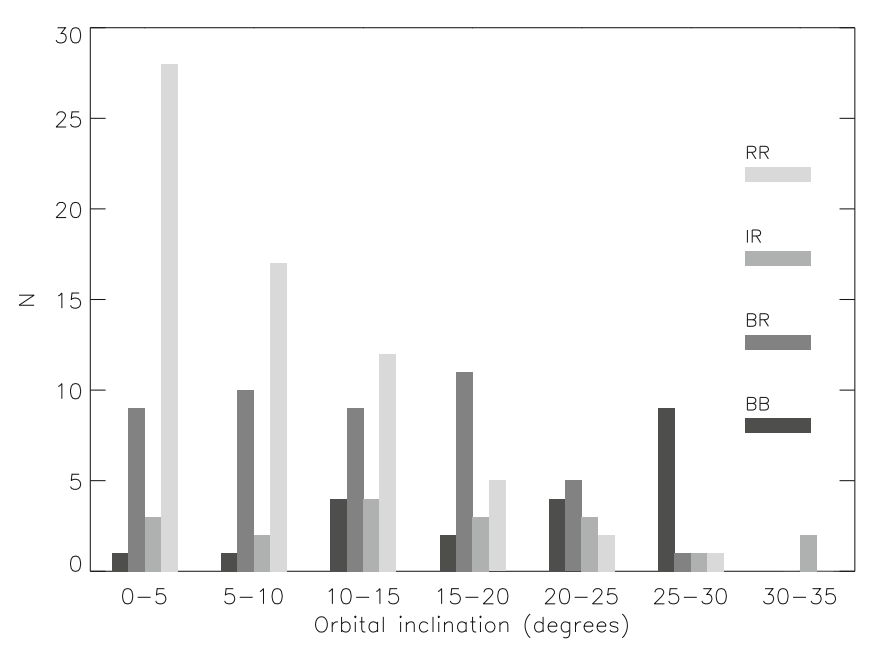

Fig. 4. Distribution of the taxa with respect to the orbital inclination of the objects. A $5^{\circ}$ binning is adopted.

dynamically "cold" population. On the contrary, BB-types seem to be concentrated at high inclinations, confirming the suggested association of these objects with the "hot" population (Levison \& Stern 2001; Brown 2001; Doressoundiram et al. 2002).

\section{Conclusions}

During the second year of an ESO large programme on TNOs and Centaurs, photometric observations of 31 objects have been carried out. From the comparison with previous works, hints of heterogeneous surfaces have been found for 4 objects (Chariklo, Sedna, $2005 \mathrm{RM}_{43}$, and $2005 \mathrm{RR}_{43}$ ).

For 28 out of the 31 observed objects we derived the taxomomic classification (within the system by Barucci et al. 2005a), using G-mode analysis. Taxonomy for ten additional objects was obtained in the framework of our programme (DeMeo et al. 2009a), for a total of 38 TNOs and Centaurs. This sample includes 19 objects which are classified for the first time ever, which constitutes about a $14 \%$ increase of the sample of 132 objects analysed by Fulchignoni et al. (2008) using the available literature.

We took into account the 151 objects that have been classified thus far to compute the average colors of the four taxonomic groups and to analyse their distribution with respect to the dynamical properties of the objects.

Looking at the distribution of the taxa within dynamical classes, the already known color bimodality (BR, RR) of Centaurs clearly emerges. Also, all of the four objects we classified as IR belong to the classical TNOs, in agreement with the finding that IR-types are concentrated in the resonant and classical dynamical classes (Fulchignoni et al. 2008). RR-types dominate the classical TNOs, but a similar division in the four taxa 
appears among the classical TNOs classified in the framework of our programme.

$\mathrm{BR}$ and RR classes dominate among the population at small $\left(a \lesssim 30^{\circ}\right)$ values of semimajor axis, while all the four taxa are well represented at greater distances from the Sun.

$\mathrm{RR}$ and BB classes are more abundant at low and high orbital inclinations, respectively, which associates these objects with the dynamically "cold" and "hot" populations.

\section{References}

Alvarez-Candal, A., Barucci, M. A., Merlin, F., et al. 2010, A\&A, in press Appenzeller, I., Fricke, K., Frtig, W., et al. 1998, The Messenger, 94, 1 Barucci, M. A., Romon, J., Doressoundiram, A., \& Tholen, D. J. 2000, AJ, 120, 496

Barucci, M. A., Belskaya, I. N., Fulchignoni, M., \& Birlan, M. 2005a, AJ, 130, 1291

Barucci, M. A., Cruikshank, D. P., Dotto, E., et al. 2005b, A\&A, 439, L1

Barucci, M. A., Brown, M. E., Emery, J. P., \& Merlin, F. 2008, in The Solar System Beyond Neptune, ed. M. A. Barucci, H. Boehnhardt, D. P. Cruikshank, \& A. Morbidelli (Tucson: Univ. of Arizona Press), 143

Bauer, J. M., Meech, K. J., Fernández, Y. R., et al. 2003, Icarus, 166, 195 Brown, M. E. 2001, AJ, 121, 2804

DeMeo, F. E., Fornasier, S., Barucci, M. A., et al. 2009a, A\&A, 493, 283

DeMeo, F. E., Barucci, M. A., Fornasier, S., et al. 2009b, A\&A, submitted

Doressoundiram, A., Peixinho, N., de Bergh, C., et al. 2002, AJ, 124, 2279

Doressoundiram, A., Peixinho, N., Doucet, C., et al. 2005, Icarus, 174, 90

Doressoundiram, A., Boehnhardt, H., Tegler, S. C., \& Trujillo, C. 2008, in The Solar System Beyond Neptune, ed. M. A. Barucci, H. Boehnhardt, D. P. Cruikshank, \& A. Morbidelli (Tucson: Univ. of Arizona Press), 91
Fulchignoni, M., Birlan, M., \& Barucci, M. A. 2000, Icarus, 146, 204

Fulchignoni, M., Belskaya, I., Barucci, M. A., de Sanctis, M. C., \& Doressoundiram, A. 2008, in The Solar System Beyond Neptune, ed. M. A. Barucci, H. Boehnhardt, D. P. Cruikshank, \& A. Morbidelli (Tucson: Univ. of Arizona Press), 181

Gaudi, B. S., Stanek, K. Z., Hartman, J. D., Holman, M. J., \& McLeod, B. A. 2005, ApJ, 629, L49

Gladman, B., Marsden, B. G., \& VanLaerhoven, C. 2008, in The Solar System Beyond Neptune, ed. M. A. Barucci, H. Boehnhardt, D. P. Cruikshank, \& A. Morbidelli (Tucson: Univ. of Arizona Press), 43

Guilbert, A., Barucci, M. A., Brunetto, R., et al. 2009, A\&A, 501, 777

Hawarden, T. G., Leggett, S. K., Letawsky, M. B., Ballantyne, D. R., \& Casali, M. M. 2001, MNRAS, 325, 563

Hudson, R. L., Palumbo, M. E., Strazzulla, G., et al. 2008, in The Solar System Beyond Neptune, ed. M. A. Barucci, H. Boehnhardt, D. P. Cruikshank, \& A. Morbidelli (Tucson: Univ. of Arizona Press), 507

Landolt, A. U. 1992, AJ, 104, 340

Leinhardt, Z. M., Stewar, S. T., \& Schultz, P. H. 2008, in The Solar System Beyond Neptune, ed. M. A. Barucci, H. Boehnhardt, D. P. Cruikshank, \& A. Morbidelli (Tucson: Univ. of Arizona Press), 195

Levison, H. S., \& Stern S. A. 2001, AJ, 121, 1730

Merlin, F., Alvarez-Candal, A., Delsanti, A., et al. 2009, AJ, 137, 315

Moorwood, A., Cuby, J.-G., Biereichel, P., et al. 1998, The Messenger, 94, 7

Peixinho, N., Doressoundiram, A., Delsanti, A., et al. 2003, A\&A, 410, L29

Perna, D., Dotto, E., Barucci, M. A., et al. 2009, A\&A, 508, 451

Persson, S. E., Murphy, D. C., Krzeminski, W., Roth, M., \& Rieke, M. J. 1998, AJ, 116, 2475

Sheppard, S. S., \& Jewitt D. C. 2002, AJ, 124, 1757

Tegler, S. C., Bauer, J. M., Romanishin, W., \& Peixinho, N. 2008, in The Solar System Beyond Neptune, ed. M. A. Barucci, H. Boehnhardt, D. P. Cruikshank, \& A. Morbidelli (Tucson: Univ. of Arizona Press), 105 